\title{
Improving Pre-Service Teachers' Visual Literacy through Online Photo-Sharing Applications
}

\author{
doi:10.3991/ijet.v6i1.1360 \\ Alaa Sadik \\ Sultan Qaboos University, Muscat, Sultanate of Oman
}

\begin{abstract}
This study aims to investigate how pre-service teachers' visual literacy skills are affected by their use of online photo management and sharing applications like Flickr. Two approaches are used to develop the visual literacy skills of pre-service teachers through Flickr. The first is to help them decode visuals through practicing analysis techniques, and interpreting and creating meaning from visual stimuli. The second is to help them encode visuals as a tool for communication. Visual literacy tests, participants' logs, photograph evaluation instruments, and interviews are used to assess the improvement in the participants' visual literacy skills. The results of the analysis revealed that the pre-service teachers' skills in interpreting, understanding, and appreciating the meaning of visual messages were enhanced through online exchange and interaction by means of photo management and sharing applications. They were able to communicate more effectively through applying the basic principles and concepts of visual design.
\end{abstract}

Index Terms-Teacher training, visual literacy; photosharing applications; Flickr

\section{INTRODUCTION}

Today's culture has become so visual that teachers and students get considerable information from visual elements. These visual elements are increasingly appearing in teaching and learning resources, delivered across a range of media in a variety of formats: textbooks, multimedia presentations, computer tutorials, television programs, and Web resources (Sims et al., 2002). Visual information usually comes in the form of line drawings, photographs, maps, diagrams, flowcharts, graphs, time lines, geometrical figures, and Venn diagrams.

Educators believe that using visual treatments helps learners to explore meanings clearly, directly, and easily, and yields positive results (Chanlin, 1998). For example, students can learn the word "spoon," but to look at a spoon communicates so much more. By looking at the spoon, students can read the history of eating, utensils, materials, civilization, culture, and habits (Bleed, 2005).

\section{A. Visual literacy, learning, and technology}

Wileman (1993) defines visual literacy as "the ability to read, interpret, and understand information presented in pictorial or graphic images" (p.114). He describes the ability to turn information into pictures as "visual thinking" (p.114). However, if literacy is reading and writing, Brill, Kim, and Branch (2000) believe that visual literacy is the ability to interpret, and generate or select images for communicating ideas and concepts. They indicated that a visually literate learner should be able to make sense of visible objects, and create static or dynamic visible objects. More specifically, Roblyer and Bennett (2001) explained that a visually literate learner should be able to:

- Interpret, understand, and appreciate the meaning of visual messages.

- Communicate more effectively through applying the basic principles and concepts of visual design.

- Produce visual messages using the computer and other technology.

- Use visual thinking to conceptualize solutions to problems (p. 9).

Roblyer and Bennett emphasized the need to develop appropriate selection, production, and evaluation strategies, and provide learners with the principles of visual communication, which can then be put into practice in a variety of settings, activities, and subject areas to improve the above visual literacy skills. Stokes (2002) agreed that students need to learn how to communicate using visual language, and know terms such as composition, foreground, and background in order to talk about images. Students need to be able to describe everything that they see and apply critical thinking to images in the same way they apply critical thinking to text.

Two major approaches have been suggested for developing visual literacy skills (Heinich et al., 1999). The first is to help learners decode (read) visuals through practicing analysis techniques. Decoding involves interpreting and creating meaning from visual stimuli. The second is to help them encode (compose) visuals as a tool for communication.

Decoding of visual messages can be improved by asking questions, such as: What does this image mean to me? What is the relationship between the image and the displayed text message? How is this message effective? Similar questions are asked during visual message creation as well: How can I visually depict this message? How can I make this message effective? What are some visual/verbal relationships I can use? (Riesland, 2005). Riesland believes that once students internalize these questions, they will not only be able to encode and decode visual messages, but will also be prepared to communicate with a level of visual sophistication that will carry them through multimedia-dependent and modern work environments.

Research has explored the relationship between learning and technology, and indicated that recent technologies like computers and the Internet have changed teaching and learning practices, and provided learners with more responsibilities and opportunities for their learning to innovatively locate and use resources to construct meaning 
(Sims et al., 2002). The World Wide Web, for example, as a graphical user interface application requires skills for recognizing navigation elements, controlling menus, and reading images in order to derive meaning from what is being communicated. In addition, photo-editing tools have made it easy for anyone to create or manipulate images, and to disseminate those images more widely than at any point in history.

In addition, the growth of image-rich resources is due in part to the wide availability of graphics software, and digital image management and sharing Web applications that facilitate the creation, storage, dissemination, and exchange of images (Sims et al., 2002). Inexpensive storage and widely available dissemination methods have made digital images a convenient and easily available information format (Chen, 1999). During the past three years, a new generation of Web-based applications for collaboration and community building has emerged, providing Web users with a tremendous capability to connect with and share a variety of resources. Among these social applications are photo-sharing applications.

\section{B. Flickr: a photo-sharing community}

While there are tens of photo-hosting applications on the Web, one of the most innovative is Flickr. Flickr is a photo management and sharing online application that provides a place to share photos and meet people with similar interests, even if photography is not their focus. Using Flickr, users can upload, download, tag, rate, and comment on photos. Users can manage and organize images, and create private or public groups to cultivate a sense of community.

Flickr offers a vast collection of images that are not available elsewhere, and its collaborative tools have made it popular in higher education. Flickr contains imagery that can be used in teaching to help develop visual literacy skills. University instructors have begun using Flickr images in their courses to share, critique, and analyze visual information. In addition, the design of Flickr intentionally promotes the development of community. The ability to engage users in a conversation about a photo, and to update that photo based on comments received, builds a sense of community.

Another key feature of Flickr is its integration with most of the major blogging services (like Blogger), which easily allows students and instructors to upload photographs into their blogs with a click of the "Blog this" button. Flickr also provides RSS feeds for everyone's photos, group and forum discussions, and specific tags so that students and teachers alike can syndicate their photos into their course Web pages. RSS feeds also allow teachers to have their students' projects delivered directly to their browsers, saving the teacher the time-consuming task of having to enter each student's URL in order to view his/her portfolios.

\section{Problem Of The Study}

Recent technologies (e.g., hypermedia applications and the Web) necessitate the need for visual literacy skills, since the effective use of these technologies requires visually literate teachers and students (Stokes, 2002). Although visual literacy can play a valuable role in the teaching and learning processes, there is a fear that visual resources can serve as decoration rather than information unless teachers have the chance to learn and teach how to

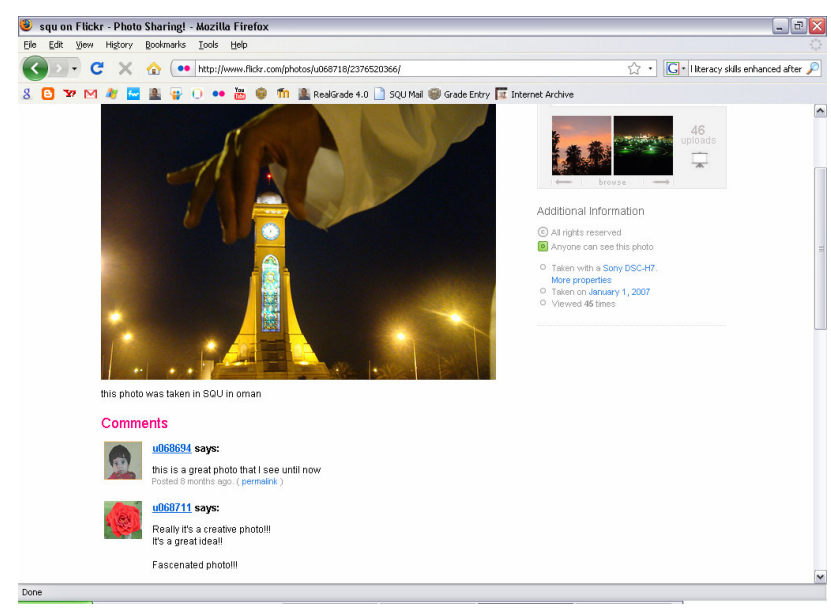

Figure 1. Flickr member's photostream page

interpret or make meaning out of these resources. Therefore, emphasis was given to the need to provide preservice teachers with a visuals-based community in which they can interpret, produce, and respond to visuals.

\section{RESEARCH QUESTIONS}

The study aims to investigate how pre-service teachers' visual literacy skills are affected by their use of online photo management and sharing applications like Flickr. Therefore, this study seeks to answer the following questions:

1. To what extent are the pre-service teachers engaged in decoding and encoding visuals through their participation in Flickr?

2. Are the pre-service teachers' visual literacy skills enhanced after using Flickr to share, communicate, and exchange visuals?

3. What are the pre-service teachers' concerns and views regarding the use of Flickr in enhancing their visual literacy skills?

\section{Purpose Of The STUdy}

The purpose of this study was to investigate the influence of Flickr, an online photo management and sharing application, on visual literacy skills when implemented in a technology course for pre-service teachers. To achieve this purpose, a series of Flickr-based activities aimed to assist pre-service teachers in interpreting, producing, thinking, and communicating visually were implanted. By the end of the study, the pre-service teachers would be able to describe the images they see and apply critical thinking to images in the same way that they apply critical thinking to text.

\section{Methodology}

Two approaches are used to develop the visual literacy skills of the pre-service teachers through Flickr: the first is to help them decode visuals through practicing analysis techniques, and interpreting and creating meaning from visual stimuli. The second is to help them to encode visuals through composing and analyzing the visual elements of visuals in a social context.

\section{PARTicipants AND PROCEDURES}

The sample consisted of pre-service teachers enrolled in an instructional technology course (TECH2113: Photog- 
raphy in Education) during the spring of 2007/2008, tutored by the researcher, at the College of Education, Sultan Qaboos University. The participants had adequate experience in computer and Internet use. The entire class $(n=28)$ was asked to participate in the study and respond to evaluation scales and interviews, and they were guaranteed confidentiality regarding their responses.

At the beginning of the study, a workshop was provided to explain the purpose of the study, the main features of Flickr, the basic concepts of visual literacy (importance, implications, syntax, and semantics), the participants' active roles, and how they will be engaged in browsing, searching, downloading, commenting, tagging, composing, editing, and uploading images to Flickr for three months. A number of discussions were held around issues in visual literacy and Flickr. The pre-service teachers were encouraged to display and share their images, and to form communities where they could exploit each other's skills and benefit from exposure to multiple work. They were also encouraged to engage in dialogue, revise images, and reach a greater level of visual communication.

\section{INSTRUMENTS}

To answer the research questions, the following four instruments were used:

\section{A. Online Tracking and Observation}

To assess the extent to which the pre-service teachers are engaged in real decoding and encoding activities through their participation in Flickr, emphasis was given to the need for in-depth analysis of the pre-service teachers' logs using online tracking and observation approaches. Since Flickr is a photo-sharing community, it seems adequate to measure engagement based on the photos a student has produced. Using the quantity of photos is less appropriate because it does not say anything about the quality and the appreciation of the community (Egger et al, 2008).

Therefore, engaged and quality content producers are identified using the following criteria: quantity and quality of images posted on Flickr, quantity and quality of comments posted on other users' photostreams, appropriateness of tags used to describe images, number of views and downloads from each participant's photostream, and sharing and exchanging of images.

To evaluate the quality of images composed and uploaded on Flickr, the Association of Texas Photography Instructors (ATPI) has developed the Photograph Evaluation Instrument to be used by judges and teachers. The instrument is composed of 5 categories (technical quality, composition, subject matter, originality, and meaning) containing 20 items on a five-point scale from excellent (5) to poor (1), with two open-ended questions for comments on the technical and educational aspects of the photograph.

The instrument uses two basic ways to judge photos. The first involves spreading out all of the entries in any particular category and judging each one against each other. The second involves judging each one against a fixed set of criteria. To determine the reliability of the instrument, face and content validity was achieved by field-testing the instrument among a sample of pre-service teachers not included in the random sample $(n=23)$. No editing or revision of the instrument was required. Using Cronbach's alpha, high reliability scores $(>.89)$ were cal- culated on the instrument from those participating in the field test.

\section{B. Visual Literacy Test}

To assess whether the pre-service teachers' visual literacy skills were enhanced via interpretation, development, and sharing of photographs or not, the need to use a visual literacy evaluation instrument was emphasized. However, reviewing the literature revealed that instruments for measuring perceptual and problem-solving ability, such as the GEFT (Weymer, 2002), require formal, laboratorybased protocols to be properly administered. This includes performance timing, strict material preparation, and compliance with administration and grading protocols. This type of instrument may intimidate university students and produce undesirable effects, such as lack of participation and confidence issues. Less formal assessment options for determining visual literacy would be preferable in university situations (Goodman, 2003).

Therefore a basic visual literacy test, empirically proven to measure the level of visual literacy a learner possessed, was developed through a review of the literature, subject matter experts and content validity in several phases using both quantitative and qualitative methods. First, the above definitions of visual literacy served as a basis for beginning to understand what the pre-service teachers need to know and be able to do in order to be visually literate. Second, a thorough review of the literature revealed that being visually literate is a combination of syntax and semantics.

According to Bamford (2003), the syntax of an image can be regarded as the pictorial structure and organization. Examples of visual syntax include scale, motion, framing, color, direction, foreground, background, shape, texture, location, and contrast. Semantics refers to the ways images fit into a cultural process of communication. Semantics include looking at the way meaning is created through form and structure, the ideas that shape the interpretation of representations and social interaction with images. Examples of questions for developing an understanding of visual semantics include: For what purpose was the image created? What has been omitted, altered, or included in an image? And what does the image say about an event?

In addition, Gardner's (1983) definition of visual intelligence, which included the ability to recognize instances of the same element, create graphics, conjure mental imagery, and transform elements, was used to form and structure the instrument items. For example, the preservice teachers could be asked to look at different versions of the same photograph, which varied on framing and simplicity, read a paragraph, and then answer the following open-ended and multiple questions: What are these pictures about? Why did you choose this picture? Select the picture that best describes the above paragraph.

In addition, students could be asked to locate, copy, and paste an image to fulfill a description, or to select the design element or design principle that is best represented by a set of pictures. The items were revised into simpler language for use with the pre-service teachers, and the first draft of the instrument consisted of five sections with a total of 21 items. A panel of five university faculty members with expertise in visual literacy, instructional media, and learning resources participated in evaluating the content validity of the test in terms of the wording of the statements and instructions, and the item relevance that 
would best measure the level of visual literacy. Based on $80 \%$ agreement among panel members, a total of 18 items were revised and retained in the preliminary instrument.

To establish face validity, a field test of the instrument was conducted, with an open-ended statement to obtain comments from the pre-service teachers regarding the clarity of the instructions, wording, and statements, and their willingness to respond to the open-ended questions. The field test responses to the instrument were very positive, and minor changes to the questions, examples, pictures, and test format were made based on the pre-service teachers' suggestions. Furthermore, the test-retest and internal consistency coefficients were calculated to determine the reliability of the instrument. Paired data were collected from a total of 23 pre-service teachers. The percents of agreement between the first and second administration of the instrument ranged from $82 \%$ to $93 \%$, with an overall average of $91 \%$.

\section{Interview}

To solicit the pre-service teachers' concerns and views regarding the use of Flickr in enhancing their visual literacy skills, emphasis was given to the need to develop a set of interview questions for individual interviews, as qualitative methods, to be asked of each teacher in order to provide consistent data. These questions were framed to determine the actual participation of the teachers.

Individual interviews, carried out during and after the three-month implementation period, were conducted in person by the researcher, and the data were analyzed to identify the teachers' concerns and views. Interviews were conducted with open-ended questions, including:

- Did you use Flickr as planned? Describe.

- What is your view on online photo management and sharing?

- What positive features of Flickr were you able to identify at the end of the implementation period?

- Do you think the online exchange of images positively influences your visual literacy skills? Describe.

- What obstacles did you encounter in using Flick? Can they be overcome?

\section{VIII.RESULTS}

The results are organized to address the research questions. In terms of the quantity of pictures posted on Flickr, assuming that every student in the class $(n=28)$ would upload at least 15 of his/her own pictures on Flicker, the total number of pictures sent to photostreams (365 pictures) represented $87 \%$ of the predicted total number of pictures (420) that would be posted. The average number of photos shared by each student through Flickr was 13.54 photos, compared with the ideal total of 15 . The majority of students posted between 10 and 21 of their own pictures, composed and edited during the course of three months.

Using only the quantity of photos to assess participation is less appropriate because it does not say anything about the quality and the appreciation of the community. To evaluate the quality of these images, the ATPI instrument was used as mentioned above. The results on technical quality showed that the majority of images had proper exposure $(71.6 \%)$, a sharp focus $(83 \%)$, and the appropriate shutter speed $(62.9 \%)$. In addition, the students se- lected good direction of light and image density in highlights or shadow $(78.4 \%)$, as well as used flash when needed (84\%).

In terms of image composition, only $23 \%$ of images were included - those with leading lines of curves or a clear center of visual interest. However, $76.6 \%$ of images were simple and filled with subjects without unwanted elements. In addition, $53 \%$ of students used the rule of thirds to provide balance within the photography by offsetting the subject from the center of the frame, and 59.3\% cropped their images properly. Overall, the students' pictures met most of the technical, composition, and subject matter evaluation criteria.

In terms of the quantity and quality of comments posted on other users' photostreams and Flickr walls, the analysis of the students' logs shows that student participation varied from no participation, to positive and regular involvement. The majority of students $(87.5 \%)$ posted between 7 and 12 messages by commenting or replying to other users' comments. Only two students did not comment or post on other users' photostreams.

In addition, qualitative analysis of the students' messages was conducted in terms of interaction, and cognitive and content-related elements. Content analysis revealed that posts that were judgment-oriented in nature made up $36 \%$ of the total number of posts. However, only $16.7 \%$ of posts were comments on messages sent by others. Of the messages, $79.9 \%$ expressed appreciation for the pictures created or posted by others.

Regarding the assumption that tags used to describe images which can make them available to Flickr users, the strategy was only to consider images matching certain tags when determining the appropriate tags. The analysis of images and their tags revealed that although many keywords were assigned specifically by the students, the use of tags for defining images was very limited, and the tags were subject to mistakes. Only $32 \%$ of the images uploaded by the students have the appropriate tags to describe their content, making them searchable by other users.

In terms of the sharing and exchanging of images as an indicator of cooperation among participants, the Flickr "Favorites" function was used to indicate successful sharing of images. The number of times the picture of a student was referenced in this way, as well as the number of views and downloads of each participant's picture used, represented some appreciation of the producer's work. The results showed that the most appreciated and popular student in the group had a Flickr FavCount of 14. In contrast, 6 students had a FavCount of zero. In addition, the average image download per student was 3.44. The top image download was 12 times (Table 1).

To investigate the effect of Flickr-based interaction and sharing activities on the pre-service teachers' visual litera-

TABLE I.

ENGAGEMENT IN FLICKR FUNCTIONS

\begin{tabular}{|l|c|c|}
\hline Function & Total & Mean \\
\hline Pictures & 365 & 13.54 \\
\hline Ongoing contacts & 46 & 2.87 \\
\hline Comments & 220 & 8.16 \\
\hline Favorites & 25 & 3.55 \\
\hline Views & 367 & 19.34 \\
\hline Downloads & 178 & 3.44 \\
\hline
\end{tabular}


acy, a pre-test/post-test design with the Visual Literacy Test was used, and free student answers were coded for quality. Overall, the results of the paired $t$-test show a significant difference between the pre-test and post-test results (Table 2).

Significant differences were evident for all visual literacy test dimensions. The results indicate that the preservice teachers were able to understand, interpret, and appreciate visual design elements (including encoding and decoding), create and manipulate images, and visualize thoughts and meaning, as shown below. The strongest improvements in visual literacy were in seeing connections between compositional elements and message (Table $3)$.

In terms of the participants' concerns and views regarding the use of Flickr, overall the participants' responses revealed that they favored the use of Flickr as a social network for sharing images over the Internet, and enjoyed reviewing and commenting on other users' posts. The majority of participants accessed Flicker 2-3 times a week during the course of three months. A student mentioned that he learned useful photography skills when he participated in Flickr. He commented: "I learned a lot of good skills working with Flickr society. I used it much more than any other photo sharing service." Another student commented, "Flickr use in this course increases what I have to learn about photos and increases my analytical skills." In addition, a student found that "Flickr is a storehouse of good pictures which can be accessed from one location." In terms of accessibility and ease of use, a student said, "Using Flickr, it is easy to find information and many types of pictures. I do not have to go to search engines."

The majority of students agreed with the statements above. They felt strongly that online photo sharing is effective for communicating with other students about course work. The students believe that Flickr is an effective tool for communication with other students and that understanding the compositional elements of pictures are enhanced by communication via Flicker.

However, many students indicated that they did not perform the specific activities described at the beginning of the course. Of these activities, the students did not use Flickr to communicate or share visuals with people outside of their class. In addition, there were some negative comments about various issues, such as the activity being a waste of time and the indirect nature of communication with classmates. Two students agreed that communication with classmates via Flickr was effective, but they did not feel it was better than communicating with them in person, particularly with the huge number of participants and images. A student commented, "Using Flickr for photo sharing is pretty overwhelmed and intimidated for quite awhile."

\section{Discussion AND CONCLUSION}

The fast-growing array of Web-based social networking applications and resources is viewed as a significant opportunity for collaboration and development in education. However, making such visual resources available for preservice teachers is not sufficient in itself to develop their visual literacy skills. If pre-service teachers are to develop visual literacy, the context in which they learn must encourage them to act as productive and analytical users.
TABLE II.

SCORES IN VISUAL LITERACY TEST

\begin{tabular}{|l|c|l|}
\hline & Pre-test & Post-test \\
\hline Mean score (\%) & 12.54 & 17.2 \\
\hline SD & 3.76 & 3.23 \\
\hline t-test & \multicolumn{2}{|c|}{$0.04^{*}$} \\
\hline${ }^{*} P<0.01$ & \multicolumn{2}{|l}{} \\
\hline
\end{tabular}

TABLE III. COMPARISON OF VISUAL LITERACY SKILLS

\begin{tabular}{|c|c|c|c|c|c|c|}
\hline \multirow{2}{*}{$\begin{array}{l}\text { Visual Literacy } \\
\text { Skills }\end{array}$} & \multicolumn{2}{|c|}{ Pre-test } & \multicolumn{2}{|c|}{ Post-test } & \multirow[t]{2}{*}{ Diff } & \multirow{2}{*}{$\begin{array}{c}t- \\
\text { test }\end{array}$} \\
\hline & Mean & SD & Mean & SD & & \\
\hline $\begin{array}{l}\text { Understand } \\
\text { the meaning } \\
\text { of visual } \\
\text { elements. }\end{array}$ & 3.08 & 0.63 & 3.65 & 0.54 & 0.53 & $*$ \\
\hline $\begin{array}{l}\text { Interpret and get infor- } \\
\text { mation from the pic- } \\
\text { tures. }\end{array}$ & 3.39 & 0.63 & 3.77 & 0.55 & 0.34 & $* *$ \\
\hline Produce visuals. & 1.57 & 0.54 & 2.11 & 0.78 & 0.62 & $* *$ \\
\hline $\begin{array}{l}\text { Select images that best } \\
\text { describe ideas and con- } \\
\text { cepts. }\end{array}$ & 2.04 & 0.70 & 2.65 & 0.48 & 0.61 & $*$ \\
\hline $\begin{array}{l}\text { Convey messages and } \\
\text { emotions through the } \\
\text { images. }\end{array}$ & 2.80 & 0.95 & 3.38 & 0.62 & 0.87 & $*$ \\
\hline $\begin{array}{l}\text { Analyze image ele- } \\
\text { ments. }\end{array}$ & 2.00 & 0.76 & 2.47 & 0.91 & 0.48 & $* *$ \\
\hline $\begin{array}{l}\text { Emotional response to } \\
\text { pictures. }\end{array}$ & 3.50 & 0.59 & 3.78 & 0.44 & 0.29 & $*$ \\
\hline $\begin{array}{l}\text { Draw conclusions from } \\
\text { pictures. }\end{array}$ & 1.59 & 0.55 & 1.98 & 0.77 & 0.62 & $*$ \\
\hline $\begin{array}{l}\text { Understand the aim of } \\
\text { the creator. }\end{array}$ & 2.77 & 0.54 & 3.21 & 0.91 & 0.89 & $* *$ \\
\hline $\begin{array}{l}\text { Understand composi- } \\
\text { tional elements, includ- } \\
\text { ing color, shape, pattern, } \\
\text { and texture. }\end{array}$ & 1.76 & 0.67 & 2.10 & 0.89 & 0.52 & $*$ \\
\hline
\end{tabular}

This activity-based approach has constructivist aims, and exploits both collaborative interaction and access to information-rich resources. The pre-service teachers' activities were supported by a range of tools that provided access to online images and allowed them to share their knowledge and skills.

The analysis of Flickr logs defined performance indicators to explore the success of the pre-service teachers. The above results show that the pre-service teachers took advantage of the social networking functions, and formed a network with respect to contact relations and group memberships. The results revealed that Flickr provided a place for the pre-service teachers to be exposed to a variety of images, critically investigate and interpret their classmates' photos, realize captions and meanings, add their comments and ask questions, and be engaged in conversations about photos. At the same time, photo creators were able to express their views and conceptualization of information; share their photos; tag their photos with keywords dealing with texture, color, and other attributes; add annotations to their photos; read and respond to the collective commentary; create photo sets and collections to manage content; and participate in topical groups to cultivate a sense of community. 
These activities offered an opportunity for the preservice teachers to interpret, understand, and appreciate the meaning of images, convey messages and emotions through images in accordance with the principles of visual literacy, and learn from each other, as reflected in the significant improvement in their visual literacy scores.

To meet the growing need for visually literate teachers, teacher education programs need to incorporate technology across the curriculum. For pre-service teachers, for whom technology is an integral part of the teacher education program, photo management and sharing applications can be used as a resource to enhance their overall visual experience, and equip them with visual literacy knowledge and skills. In this way, they can create effective visual instructional materials, and pass on their knowledge and skills in visual literacy to their students, the next generation.

For in-service teachers, Flickr can provide a community accessible whenever and wherever they need assistance in visual areas. It transcends the walls of isolation that separate teachers and extends learning opportunities without the teachers having to leave their school buildings, particularly with the numerous number of multimedia applications and technology available in schools.

\section{REFERENCES}

[1] A. Bamford. "The visual literacy white paper". Adobe Systems Pty Ltd, Australia, 2003.

[2] R. Bleed. Visual literacy in higher education. EDUCASE Learning Initiative. http://net.educause.edu/ir/library/pdf/eli4001.pdf, 2005.

[3] M. Brill, D. Kim, \& M. Branch, Visual literacy defined: the results of a Delphi study-can IVLA (operationally) define visual literacy? In Griffen, R., Williams, V. \& Lee, J. (Eds.), Exploring the visual future: art design, science and technology. Blacksburg, VA: The International Visual Literacy Association. 9-15, 2001.

[4] L. Chanlin , Animation to teach students of different knowledge levels. Journal of Instructional Psychology, vol. 25, no. 3, pp. 166-175, 1998.

[5] H. Chen, Intellectual access to images: image database systems. Library Trends, 48(2), 291-302.
[6] M. Egger, K. Fischbach, P. Gloor, A. Lang, M. Sprenger, How to Identify Successful Actors of the Flickr Community and How to Determine Their Attributes. GI Jahrestagung, 2, 949-954. http://digitale-soziale-netze.de/papers, 2008.

[7] H. Gardener, Frames of mind: The theory of multiple intelligences. New York: Basic Books, 1983

[8] P. Goodman, Pictionary: a test for visual literacy. In D. Lassner \& C. McNaught (Eds.), Proceedings of World Conference on Educational Multimedia, Hypermedia and Telecommunications 2003, 2370-2373. Chesapeake, VA: AACE, 2003

[9] R. Heinich, M. Molenda, D. Russell, \& E. Smaldino, Instructional media and technologies for learning (6th ed.). Upper Saddle River, NJ: Prentice-Hall, 1999.

[10] E. Riesland, Visual Literacy and the Classroom. New Horizons for Learning. http://www.newhorizons.org, 2005

[11] D. Roblyer, \& K. Bennett, The fifth literacy: Research to support a mandate for technology-based visual literacy in pre-service teacher education. Journal of Computing in Teacher Education, vol. 17, no. 2, pp. 8-15, 2001.

[12] E. Sims, R. O'Leary, J. Cook \& G. Butland, Visual Literacy: What is it and do we need it to use learning technologies effectively? Proceedings of ASCILITE 2002, pp. 885-888, 2002.

[13] S. Stokes, Visual literacy in teaching and learning: A literature perspective. Electronic Journal for the Integration of Technology in Education, vol. 1, no. 1, 2002. http://ejite.isu.edu/Volume1No1/Stokes.html. Accessed 20 October 2007.

[14] R. Weymer, Factors Affecting Students' Performance in Sixth Grade Modular Technology Education. Journal of Technology Education, vol. 13, no. 2, 2002, http://scholar.lib.vt.edu/ejournals/JTE/v13n2/weymer.html .

[15] E. Wileman, Visual communicating. Englewood Cliffs, N.J.: Educational Technology Publications, 1993.

\section{AUTHOR}

A. Sadik is an associate professor of instructional technology at South Valley University, Egypt, and currently work at Sultan Qaboos University, Oman (e-mail: alaasadik@gmail.com).

Manuscript received 23 June 2010. Published as resubmitted by the author March 1 $1^{\text {st }}, 2011$. 